\title{
MODEL TIME-FREQUENCY RESOURCE ALLOCATION WIMAX AIMED AT IMPROVING THE ELECTROMAGNETIC COMPATIBILITY
}

\author{
${ }^{1}$ A. V. Lemeshko and ${ }^{2}$ S. V. Garkusha \\ ${ }^{1}$ Kharkiv National University of Radio Electronics, Kharkiv, Ukraine \\ E-mail: avlem@mail.ru \\ ${ }^{2}$ Poltava University Economics and Trade, Poltava, Ukraine \\ E-mail: sv.garkusha@mail.ru
}

\begin{abstract}
The results of development a mathematical model for capacity allocation downlink technology WiMAX. The novelty of the proposed model is possible preventive limit transmission rate allocated to the service flows of the user stations in the downlink by using the WiMAX technology linear or linear-quadratic objective function. It is shown that the use of a linear-quadratic objective function in comparison with the linear, can produce a more equitable management of requests based on the relative priorities. The analysis of known methods for dividing the time-frequency resource WiMAX technology showed that they all focus on the distribution between the subscriber stations all bandwidth downlink. The model proposed is directed to the allocation of each user station a guaranteed bit rate in the absence of overload downlink as well as the preventive rate limiting allocated user stations under overload conditions. Using the mathematical model is directed to distribution between subscriber stations of a time-frequency resource of the downlink, which in turn improves the conditions in the electromagnetic frequency range used. The influence of the priority request rate used in the model, the nature of the possible failures.
\end{abstract}

Keywords: WiMAX, time-frequency resource, the electromagnetic compatibility, mathematical model, guaranteed bit rate.

\section{INTRODUCTION}

At present time while using wireless telecommunications networks (WTN) particularly networks of WiMAX there is a problem of providing electromagnetic compatibility of the networks in distinguished diapason of frequencies. Besides that under the conditions of heterogeneity and multiprotocol of modern WTN especially at overload and limitation of frequency and time resources there is a problem of increasing the level of coordination for task decision of distribution the downlink bandwidth between subscriber station (SS) and limitation traffic intensity in the network $[1,2]$. There should be taken into account possibility of presenting to every service flow (SF) at MAC sublevel the data link level network with required quality of service (QoS). Considering multiservice character of modern WTN, refusals of service should refer to first of all background traffic.

The analysis showed that know methods of distribution of frequency and time recources of downlink in WiMAX use Best Effort. The quality is not guaranteed, but between SS all the available frequency and time resources is distributed [2]. However in WiMAX besides Best Effort supported several classes of service $(\mathrm{CoS})$ among which Not Real Time, Real Time,
Extended Real Time, Unsolicited Grant Service, directed upon working out guaranteed bandwidth.

So, urgent scientific and practical task is developing and researching mathematic models and methods of distributing frequency and time resources of downlink WiMAX, guaranteeing their giving to SSs and their SFs required bandwidth to be the basis of promising technological decisions. Developing the model and methods must be directed upon giving only the necessary quantity of frequency and time resources, that will favor the improvement of electromagnetic environment in the used frequency diapason. The result is developed mathematic model of downlink WiMAX bandwidth distribution.

\section{MODEL OF FREQUENCY AND TIME RESOURCE DISTRIBUTE IN DOWNLINK}

While solving the task of data burst scheduling for SFs of all SSs transmitting in downlink within the suggested model it is necessary to provide Boolean managing variable calculation $\left(x_{k, m}^{n}\right)$, determining the order of slots distribution:

$x_{k, m}^{n}=\left\{\begin{array}{l}1, \text { if the } m-\text { th slot is used in the } k-\text { th } \\ \text { subchannel allocated by the } n-\text { th SS; } \\ 0, \text { otherwise. }\end{array}\right.$ 
Besides that there is magnitude $\alpha^{n}$, modelling part of necessary bandwidth allocation refusals [3]. Then vector of the searched parameters considering (1) can be presented in the form:

$$
\vec{X}=\left[\begin{array}{c}
x_{k, m}^{n} \\
--- \\
\alpha^{n}
\end{array}\right] .
$$

Coordinates of vector $\vec{X}$ is multitude of variables $x_{k, m}^{n}$, where $n=\overline{1, N}, m=\overline{1, M}, k=\overline{1, K}$, and range of parameters $\alpha^{n}$, where $n=\overline{1, N}$. At this multitude of variables $x_{k, m}^{n}$ will be determined by $N \times M \times K$, where $N$-common number of downlink, transmitted by all SSs, $M$ - number of slots at one sub-channel of downlink for useful information transmitting, $K-$ number of sub-channels in frequency channel and number of parameters $\alpha^{n}$ will be equal to $N$.

According to results of variables (2) calculation there is sub-channel fastening and distributing after SS to transmit primary data in downlink. Besides that at calculation the vector of searched parameters $\vec{X}$ it is necessary to fulfil a range of conditions/constraints:

1) Condition of $k$-th sub-channel fastening during transmitting $m$-th slot no more than after one service flow.

2) Condition of $n$-th service flow number of slots fastening, providing necessary bandwidth at modulation and coding scheme (MCS) used by corresponding SS.

3) Condition of one burst for $n$-th service flow corresponded SS to minimize number of slots allocated for service information transmitting.

4) Condition of "rectangular" burst forming corresponding technological particularities of IEEE 802.16 family standards, using OFDMA.

5) Condition of necessary slots number for service information transmitting reserving.

According to physics of the task upon coordinates $\alpha^{n}$ of vector $\vec{X}$ there put on the next limitations [3]:

$$
0 \leq \alpha^{n} \leq 1
$$

or

$$
\alpha^{n} \in\{0,1\},
$$

if at the basis of agreement about Service Level Agreement (SLA) acceptable (3) or acceptable (4) partial limitation of the required transmission rate.

Calculation of the searched variables (2) according to conditions/constraints better to commit while optimization task decision, providing minimum linear (5) or linear-quadratic (6) objectives functions [3]:

$$
\min _{X} C^{t} \vec{X}=\min _{X} \sum_{k=1}^{K} \sum_{m=1}^{M}\left(\sum_{n=1}^{N} c_{k, m}^{n} x_{k, m}^{n}+\sum_{n=1}^{N} c^{n} \alpha^{n}\right),
$$

$$
\min _{X}\left[\frac{1}{2} \vec{X}^{t} H \vec{X}+C^{t} \vec{X}\right],
$$

characterizing relative cost of bandwidth at the stage of burst data scheduling. Coordinates of vector $\vec{C}$ and matrix $H$ can be presented as the following:

$$
\begin{gathered}
\vec{C}=\left[\begin{array}{c}
c_{k, m}^{n} \\
--- \\
c^{n}
\end{array}\right] \quad(k=\overline{1, K} ; m=\overline{1, M} ; n=\overline{1, N}), \\
H=\left\|\begin{array}{|cccccc}
\mu c_{11}^{1} & 0 & 0 & 0 & 0 & 0 \\
0 & \mu c_{12}^{1} & 0 & 0 & 0 & 0 \\
0 & 0 & \cdots & 0 & 0 & 0 \\
0 & 0 & 0 & \mu c_{k, m}^{n} & 0 & 0 \\
0 & 0 & 0 & 0 & \cdots & 0 \\
0 & 0 & 0 & 0 & 0 & \mu c^{n}
\end{array}\right\|
\end{gathered}
$$

Coordinates of vector $\vec{C}$ determines magnitude of specific fine for using downlink slots $\left(c_{k, m}^{n}\right)$ and for limitation subscribers traffic $\left(c^{n}\right)$, under the condition that $c_{k, m}^{n} \ll<c^{n}, \mu$ - coefficient determining how many times coordinates of diagonal matrix $H$ more (less) than vector $\vec{C}$ coordinates.

\section{ANALYSIS THE RECEIVED DECISIONS}

There was also conducted analysis of downlink data burst scheduling process at joint maintenance of two SFs by guides of one SS with different priorities driven by changing relations meaning $\Delta C=c_{k, m}^{n} / c^{n}$. During the research we found out that the character of possible refusals at the suggested model using depends, first of all, on number of subscribers stations and MCS use by the stations. Secondly the character of possible refusals depends of relation of costs for loading the downlink $\left(c_{k, m}^{n}\right)$ to costs for service limitation subscribers traffic $\left(c^{n}\right)$.

Below at the fig. 1 and fig. 2 presented dependences of refusals share at allocation necessary bandwidth of high priority $\left(\alpha^{1}\right)$ and low priority $\left(\alpha^{2}\right)$ services flows for linear and linear-quadratic objects functions. The results of the analysis showed that within suggested model service of SSs requirements is realized on the base of absolute priorities. So, using linear objective function in case when sum of requirements for demanded transmission rate exceeds bandwidth of downlink so preventive limitation gets requirement from low priority SS up to full refusal of access (fig. $1 \mathrm{~b}$ ). Requirement for transmission rate from service flow with higher priority won't be jammed until there is possibility of refusal to low priority requirement (fig. 1 a). 


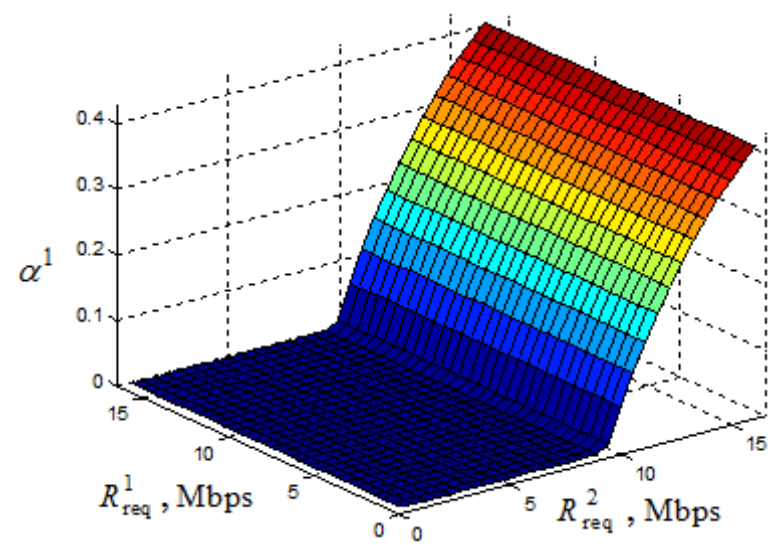

a) refusals of high priority requirement

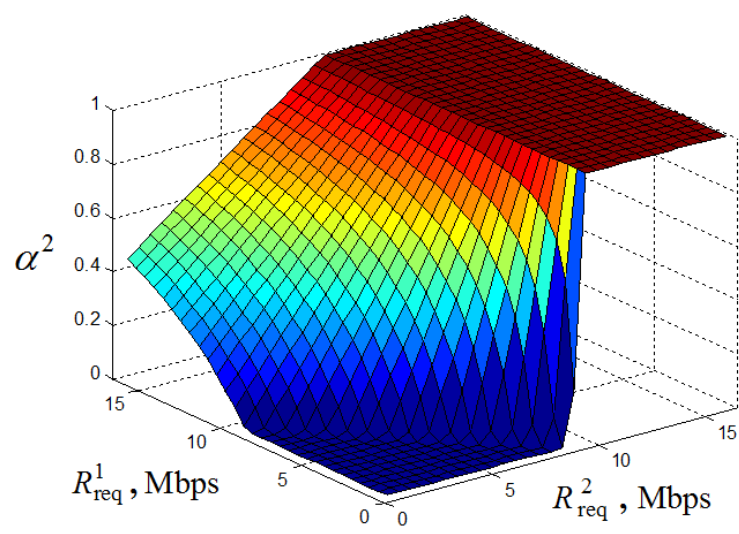

b) refusals of low priority requirement

Fig. 1. Dependence of refusals share from their requirements for demanded transmission rate at linear objective function.

Using linear-quadratic objective function there is organized more fair management at the basis of relative priorities than when using linear objective function. I.e. in case of possible overload service refusals touch upon all the services flows at this high priority has less degree (fig. 2 a) and low priority has bigger degree (fig. 2 b). Under this model has possibility to adjust preventively of traffic limitation, going into the network by changing weight coefficients also by degree of percent correlation of possible refusals magnitude when serving high priority traffic relatively to low priority by changing meaning of $\mu$ of diagonal matrix $H$.

\section{REFERENCES}

1. Garkusha S., Ahmed H. Abed Slot Allocation Model and Data Burst Scheduling in Downlink WiMAX Technology // Proc. of "12th International Conference the Experience of Designing and Application of Cad Systems in Microelectronics" CADSM'2013. Polyana, 2013. - P. 97-100.

2. Garkusha S.V. The planning model of data burst in technology WiMAX // Proceedings of the North Caucasus branch of the Moscow Technical University of Communications and Informatics.

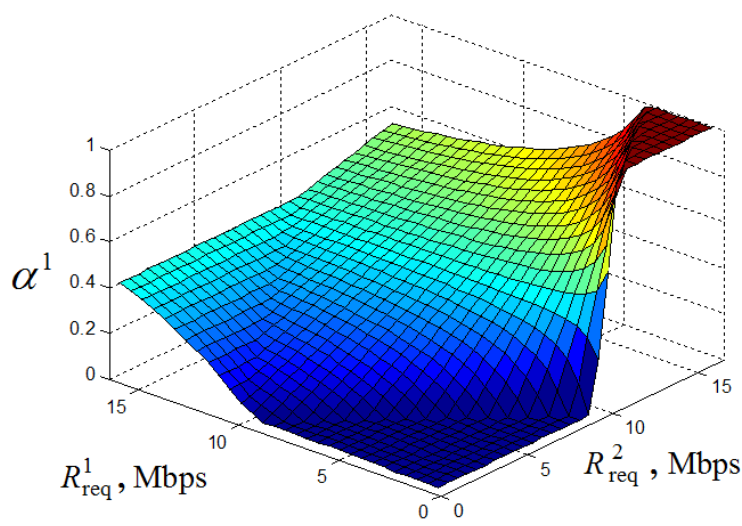

a) refusals of high priority requirement

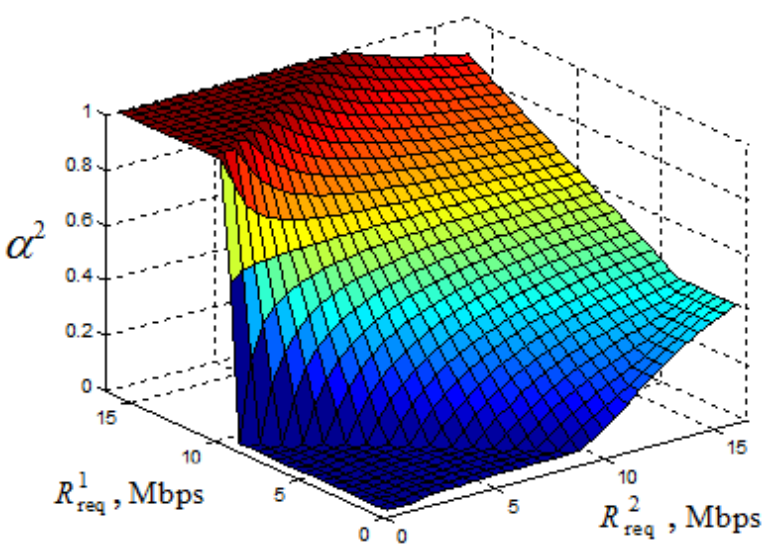

b) refusals of low priority requirement

Fig. 2. Dependence of refusals share from their requirements for demanded transmission rate at linear-quadratic objective function.

Rostov-on-Don: HRC "University" GFR MTUCI, 2013. - P. 74-77.

3. Lemeshko A.V. Results of the study models traffic management taking into account the priorities defined for multiproduct and multi-polar cases // telecommunications problems. № 2 (2), 2010. - P. 33-41. 